\title{
Ferramenta para Extração de Múltiplas Características em Exames Médicos por Imagem
}

\author{
Adriana N. Reis ${ }^{1}$, Rodrigo F. Rönnau ${ }^{2}$, Nicolas Pohren ${ }^{1}$, Jonata Becker ${ }^{1}$, Anderson \\ R. Spaniol'1, Marta R. Bez ${ }^{1}$, Rodrigo R. V. Goulart ${ }^{1}$ \\ ${ }^{1}$ Universidade Feevale - Novo Hamburgo (RS) - Brasil \\ ${ }^{2}$ Universidade do Vale do Rio dos Sinos (UNISINOS) - São Leopoldo (RS) - Brasil \\ \{adriana.anreis, rodrigo.ronnau, nicolaspohren, jonatabecker, \\ andersonspaniol1994, martabez, rodrigo.goulart\}@gmail.com
}

\begin{abstract}
Diagnostic imaging has contributed significantly to patient care, especially the use of computed tomography images, which allow the internal visualization of the body with high anatomical detail. To assist the physician in the analysis of these images, different computational solutions are found. However, it is common for each tool to perform the extraction of a specific characteristic. In this sense, the project described in this paper proposes building a tool to extract different characteristics in an integrated way, giving the doctor the support of algorithms with different purposes from the same exam. To do so, the Software Product Lines approach is adopted.
\end{abstract}

Resumo. O diagnóstico por imagem tem contribuído significativamente para o atendimento ao paciente, destacando-se o emprego das imagens de tomografia computadorizada, que permitem a visualização interna do corpo com elevado detalhamento anatômico. Para auxiliar o médico na análise dessas imagens são encontradas diferentes soluções computacionais. Porém, é comum que cada ferramenta realize a extração de uma característica específica. Assim, o projeto apresentado neste artigo propõe a construção de uma ferramenta para extração de diferentes características de modo integrado, oportunizando ao médico a execução de algoritmos com diferentes propósitos a partir de um mesmo exame. Para tanto, Linhas de Produto de Software é adotada.

\section{Introdução}

Na rotina de realização de diagnósticos a partir de imagens médicas, é responsabilidade de um especialista, ou grupo de especialistas, realizar a análise e interpretação de um exame, avaliando todas as imagens que o compõem, uma a uma. Além disso, em diversos casos, a interpretação realizada tem caráter subjetivo, uma vez que o profissional analisa as imagens de acordo com sua experiência e conhecimento.

Para auxiliar neste processo existem diferentes soluções computacionais, tanto na esfera acadêmica como ferramentas comerciais. Contudo, percebe-se que elas atendem, geralmente, a um propósito específico, tal como a segmentação de uma estrutura anatômica ou a quantificação de um determinado elemento. O que na prática não se torna atrativo aos médicos, em função da não existência de uma visão integrada das características que são processadas automaticamente. 
Assim, o projeto apresentado neste artigo tem como objetivo a construção de uma ferramenta para a extração e apresentação de diversas características em exames médicos por imagem, a partir de uma arquitetura modular e expansível. Desse modo, é esperado que ela seja utilizada pelo médico como uma plataforma única para suporte à decisão clínica, permitindo a realização de laudos mais completos e precisos.

Na próxima seção é apresentada a arquitetura de software em desenvolvimento para a construção da ferramenta, seguida dos resultados preliminares, na seção 3. Por fim, a seção 4 apresenta Considerações Finais sobre o trabalho em andamento.

\section{Arquitetura em Desenvolvimento}

A partir do problema apresentado, este estudo investiga meios para aumentar a acurácia do diagnóstico baseado em exames médicos por imagem, especificamente de tomografia computadorizada (TC), utilizando técnicas de processamento digital de imagens. Assim, a partir da execução de diferentes algoritmos, detalhados em [Reis et al. 2016], pretende-se fornecer ao médico múltiplas características que o auxiliem na tomada de decisão que envolve a elaboração de laudos. Entre as informações obtidas, estão algoritmos para: segmentação do pericárdio, segmentação dos pulmões, quantificação da calcificação arterial coronariana, e quantificação da gordura epicárdica.

Uma vez que a proposta está baseada na integração de diferentes algoritmos, identificou-se a oportunidade de aplicar a abordagem de Linhas de Produto de Software (Software Product Lines - SPL) como estratégia para a construção da ferramenta. SPL pode ser entendido como um conjunto de sistemas de software que possuem características compartilhadas, para atender necessidades de um segmento específico a partir de um núcleo comum [SEI 2017]. Assim, é possível construir produtos distintos a partir dos mesmos blocos de construção, reduzindo o seu custo de desenvolvimento e aumentando a qualidade e consistência do sistema entregue [Linden et al. 2007].

De forma geral, SPL é uma alternativa para trabalhar com famílias de produtos relacionados a uma mesma área [Wazlawick 2013]. Para implementá-la, três atividades são consideradas essenciais: desenvolvimento do núcleo de ativos de produtos, desenvolvimento de produtos e gerência [Northrop 2004]. Na fase atual do projeto, as duas primeiras estão em desenvolvimento.

O desenvolvimento dos ativos de produtos está consolidado em um framework, construído a partir de uma série de soluções desenvolvidas separadamente para processamento de imagens de TC do tórax [Reis et al. 2016], o qual é representado de forma esquemática na Figura 1. Ele está organizado em cinco módulos, descritos a seguir:

- PDI: implementações de algoritmos de processamento de imagens, que são aplicados aos exames médicos no processo de extração de características, como, por exemplo: histograma, limiarização, remoção e redução de ruídos, operações de convolução, métodos de classificação, entre outros;

- Uso Comum: classes de uso comum por todos os outros módulos, utilizadas na troca de informações entre estes; 
- Acesso a Arquivos: camada de acesso a arquivos DICOM (Digital Imaging and Communications in Medicine), que é o formato padrão dos exames utilizados;

- Orquestração do Fluxo de Processamento: camada que permite determinar a sequência de invocação dos métodos do módulo de PDI de acordo com cada característica que se deseja obter;

- Interface de Uso: interface de operação do sistema, construída sobre o padrão MVC (Model View Controller).

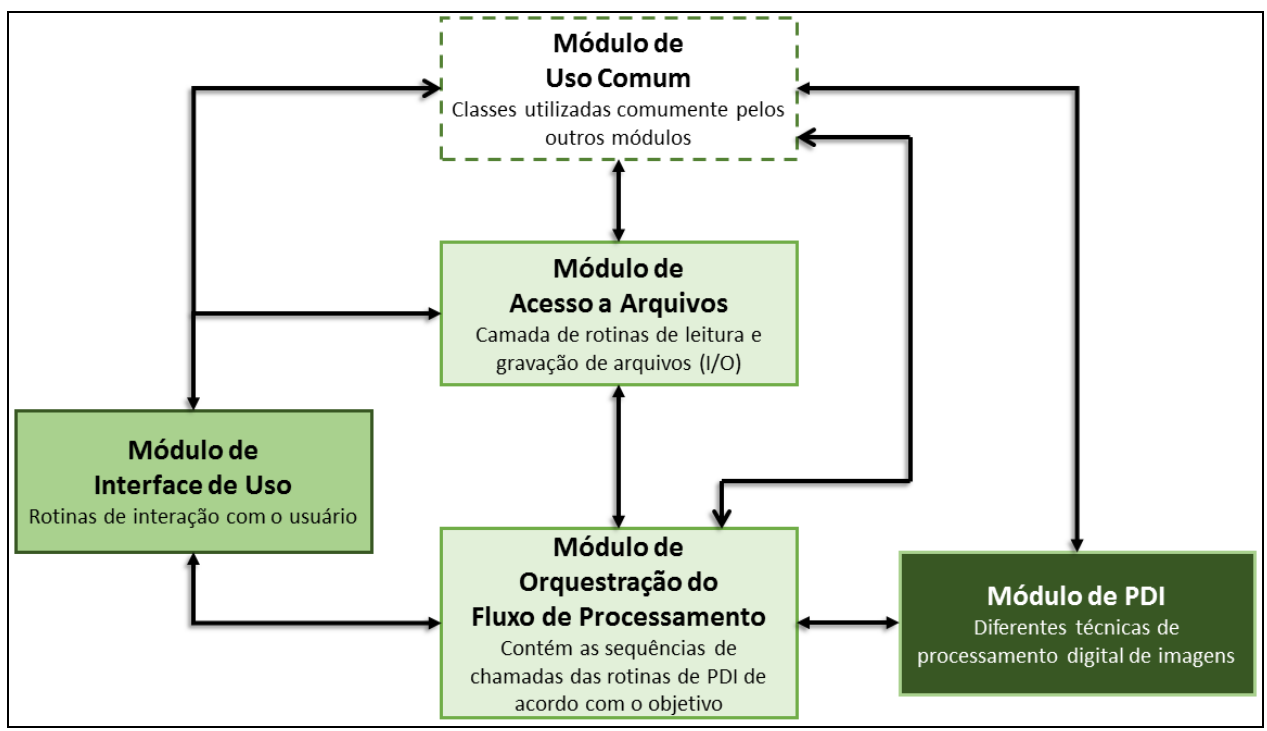

Figura 1. Diagrama simplificado do framework proposto. (Do autor, 2017)

Com base nestes módulos, as características extraídas são disponibilizadas por uma ferramenta proposta para a visualização das análises, a qual é o primeiro produto desenvolvido pelo projeto. O detalhamento deste produto é apresentado na seção a seguir.

\section{Resultados Preliminares}

A Figura 2 apresenta a interface gráfica do primeiro produto em desenvolvimento. Nela, todos os passos do processamento são executados de forma automática, sem a necessidade de intervenção humana.

Como pode ser visto na Figura 2, ao final da execução dos processos são disponibilizados os dados obtidos pela ferramenta, que também contêm recursos de visualização e manipulação das imagens que compõem os exames. Em relação à manipulação e visualização, como os exames de TC são formados, usualmente, por um grande número de imagens, é necessário permitir navegar entre elas de maneira sequencial. Além disso, estão disponíveis opções para modificar parâmetros de nível e largura de janela, possibilitando ao operador realizar ajustes finos que melhoram o contraste das diferentes estruturas anatômicas a serem avaliadas.

A validação será feita por profissionais da saúde, que irão utilizar o protótipo com exames de TC obtidos através do TCIA (The Cancer Imaging Archive), avaliando a precisão, usabilidade e contribuição da ferramenta no processo de diagnóstico. 


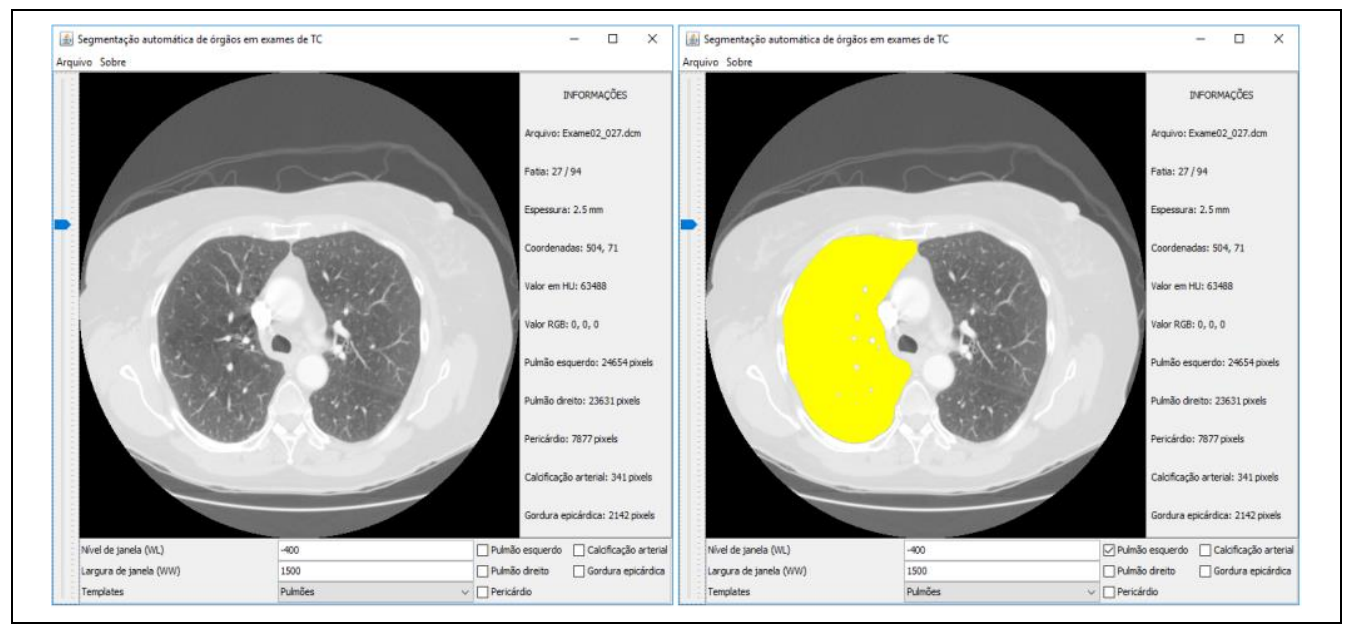

(a)

(b)

Figura 2. Visão preliminar da interface gráfica da ferramenta. Em (a) o resultado do carregamento de um exame. Em (b) o resultado do algoritmo de segmentação dos pulmões, com a opção de destaque de um deles utilizada. (Do autor, 2017)

\section{Considerações Finais}

Foi descrita a proposta da construção de uma ferramenta para a extração e apresentação de diferentes características em exames médicos por imagem, a partir de uma arquitetura modular e expansível. $\mathrm{O}$ trabalho está em fase de desenvolvimento, sendo o módulo de PDI o que está no estágio mais avançado. A interface de uso está funcional, porém estão sendo feitas pesquisas quanto ao seu design, com o objetivo de melhorar a usabilidade para profissionais da saúde. O processo de segmentação dos pulmões, que é descrito no módulo de orquestração do fluxo de processamento, está concluído, utilizando também o módulo de acesso a arquivos DICOM para obter dados dos exames que estão armazenados nesse formato.

Os próximos passos abrangem a construção dos processos de segmentação do pericárdio, quantificação de gordura epicárdica e quantificação da calcificação arterial coronariana no módulo de orquestração do fluxo de processamento. Também está prevista a expansão do módulo de acesso a arquivos, permitindo a obtenção de mais informações dos exames.

\section{Referências Bibliográficas}

Linden, F., Schmid, K. and Rommes, E. (2007), Software Product Lines in Action, Springer.

Northrop, L. (2004), Software Product Adoption Roadmap, Pittsburg: SEI.

Reis, A. et al. (2016), Ferramenta para apoio ao diagnóstico baseada em processamento de imagens de tomografia computadorizada do tórax, ERCAS 2016.

SEI - Software Engineering Institute (2017) "Software Product Lines", http://www.sei.cmu.edu/productlines/, Março.

Wazlawick, R. S. (2013), Engenharia de software: conceitos e práticas, Elsevier. 